\title{
Refractive index measurements of single, spherical cells using digital holographic microscopy
}

\author{
Mirjam Schürmann ${ }^{1}$, Jana Scholze ${ }^{1}$, Paul Müller ${ }^{1}$, Chii J. Chan ${ }^{2}$, \\ Andrew E. Ekpenyong ${ }^{1,3}$, Kevin J. Chalut ${ }^{2,4}$, and Jochen Guck ${ }^{1,2}$ \\ ${ }^{1}$ Biotechnology Center, Technische Universität Dresden, Tatzberg 47/49, 01307 Dresden, Germany \\ ${ }^{2}$ Cavendish Laboratory, Department of Physics, University of Cambridge, JJ Thomson Ave, \\ Cambridge CB3 OHE, UK \\ ${ }^{3}$ Department of Physics, Creighton University, 2500 California Plaza, Omaha, NE 68178, USA \\ ${ }^{4}$ Wellcome Trust/Medical Research Council Stem Cell Institute, Tennis Court Road, Cambridge CB2 1QR, UK
}

\begin{abstract}
In this chapter, we introduce digital holographic microscopy (DHM) as a marker-free method to determine the refractive index of single, spherical cells in suspension. The refractive index is a conclusive measure in a biological context. Cell conditions, such as differentiation or infection, are known to yield significant changes in the refractive index. Furthermore, the refractive index of biological tissue determines the way it interacts with light. Besides the biological relevance of this interaction in the retina, a lot of methods used in biology, including microscopy, rely on light-tissue or light-cell interactions. Hence, determining the refractive index of cells using DHM is valuable in many biological applications. This chapter covers the main topics which are important for the implementation of DHM: setup, sample preparation and analysis. First, the optical setup is described in detail including notes and suggestions for the implementation. Following that, a protocol for the sample and measurement preparation is explained. In the analysis section, an algorithm for the determination of the quantitative phase map is described. Subsequently, all intermediate steps for the calculation of the refractive index of suspended cells are presented, exploiting their spherical shape. In the last section, a discussion of possible extensions to the setup, further measurement configurations and additional analysis methods are given. Throughout this chapter, we describe a simple, robust, and thus easily reproducible implementation of DHM. The different possibilities for extensions show the diverse fields of application for this technique.
\end{abstract}

Keywords. digital holographic microscopy, cellular refractive index, quantitative phase microscopy, biomedical imaging, marker-free imaging

Copyright. Reproduced from Methods in Cell Biology Volume 125, Chapter 9 (identical title and authors), Pages 143-159, Copyright (2015), http://dx.doi.org/10.1016/bs.mcb.2014. 10.016 with permission from Elsevier. 


\section{Introduction}

Digital holographic microscopy (DHM) is a quantitative phase microscopy technique which allows the measurement of the phase shift introduced by an object in an interferometer. Other common imaging techniques based on interferometry, such as phase contrast (PC) or differential interference contrast (DIC) microscopy, use optical path differences to image phase variations in samples that are otherwise barely visible in bright-field microscopy. In contrast, DHM is used to measure phase changes quantitatively. The measured phase contains two sample-specific characteristics, the thickness and the refractive index (RI).

There are different approaches to use the gained information in a biological context. The determined phase can be used as a combined measure for thickness changes and RI changes of cells. For example, in the case of volume changes, the thickness as well as the RI is altered. These two quantities cannot be separated just by taking the phase into account. To study RI or thickness separately, either of the two parameters has to be known. In the course of this chapter, a method will be described on how to determine the RI of spherical cells. However, the principle stated here can also be adapted to the analysis of other samples of known shape. One of the three parameters phase, RI or thickness can then be used as output parameter of DHM measurements.

In several studies DHM has been successfully utilized to quantify cellular function. Taking the phase into account, Pavillon et al. found distinctive features in DHM measurements of mouse cortical neurons they attribute to cell volume regulation as an early indication of cell death [1. Determining the RI from the measured phase values, Chalut et al. found lineage specific differences of the RI during the differentiation of a myeloid precursor cell line [2]. In a further example, a decrease in the RI of Salmonella-infected macrophages has proven to be a reliable read-out for host-pathogen interactions [3]. Besides using the RI as a measure of cellular function, the acquired information can also be integrated into other biophysical techniques that directly depend on the RI of the sample. One example is the measurement of optically induced stress in the context of optical traps. In any trap geometry where optical forces act on a cell, as in the case of an optical stretcher, the measured mechanical response depends on the RI of the cell and therefore needs to be evaluated separately [4]. Another field of application is the interpretation of light scattering by tissues, aiming for example at marker-free sensitivity to tissue alteration in dysplasia. Here, the RI of the cells is one parameter influencing the analysis of the scattered light field [5, 6]. Furthermore, studies on retinal tissue must deal with light-tissue interactions, where the optical properties of the tissue play a crucial role. Studies on photoreceptor nuclei revealed their ability to focus transmitted light [7, 8]. The ability to act like a lens is highly dependent on the RI distribution inside these cells. All of the mentioned examples show that the RI of a cell and its distribution is a highly relevant quantity in certain areas of biology and biotechnology.

Early measurements of the RI of living cells used RI matching of the measured object with the surrounding medium [9]. In these measurements, the RI of cells in suspension is determined by adaption of the RI of the medium to that of the cells. Index matching is achieved by gradually changing the RI of the medium until the contrast in PC images between the cells and the medium is minimized. The RI of the medium corresponds to the $\mathrm{RI}$ of the cells at the point of a reversal in sample contrast. Refractive index matching is usually performed with several cells inside the field of view leading to an average RI value 
for a cell population. However, this method does not allow for an accurate determination of RI on a single cell level and it is time consuming. Quantitative phase microscopy overcomes these technical constraints and allows measuring the RI of single cells.

There are different methods to measure the phase retardation of light transmitted through an object. A common concept, also used in DHM, is light interferometry which effectively measures the phase difference induced by a sample. The optical path difference at each position is the product of the thickness of the sample and the average RI of this path. Hence, knowing the thickness of the sample at each position is a prerequisite for the determination of its RI. There have been several attempts in the past to address this problem, for example by exchanging the medium surrounding the cells [10] or measuring with two different wavelengths [11]. However, this involves rather elaborate sample preparation or setup construction. A different approach to separate the refractive index from the thickness of the sample is the combination of phase measurements with confocal microscopy. Confocal z-stacks from fluorescently labeled cells are used to determine the height at certain sample positions and the phase is measured at the same position in a subsequent measurement step [12]. Nevertheless, due to the z-stacking and the related post processing, this technique is time-consuming. Furthermore, the fluorescence labeling of the cells contradicts the maker-free nature of quantitative phase imaging. A simpler solution exists when the samples are transparent or semi-transparent spherical dielectric objects such as spherical cells. In this case the height at each position can be calculated from the determined radius of the cell in the field of view [13. With this knowledge the average RI at each lateral position of the cell can be calculated. This approach will be elaborated further later in this chapter.

In the next section, a basic version of the DHM setup will be described in detail, followed by a protocol for preparation of spherical cells in suspension. After a thorough guidance through the analysis method, possible extensions or alternatives will be addressed in the discussion part.

\section{Setup}

The basic concept of digital holographic microscopy is to measure the phase change induced by a sample in an interferometer. In one of the light paths, the so-called object beam, light is transmitted through the sample which leads to a characteristic alteration of the wavefront. The second path serves as a reference for the measurement of the phase retardation and is accordingly termed reference beam (Figure 1). The resulting interference pattern is recorded by a complementary metal-oxide-semiconductuor (CMOS) camera.

The light source is a HeNe-laser $(\lambda=632.8 \mathrm{~nm}$, HNL050L-EC, Thorlabs GmbH, Dachau, Germany) with a power of about $0.5 \mathrm{~mW}$. A non-polarizing beam-splitter cube (BS1) is used to split the beam with a ratio of about 50:50 into object and reference beam. The cells in solution are mounted horizontally on a microscope slide. Allowing for lateral movements in the field of view, the microscope slide is placed on a two dimensional translation stage. The object beam is first steered over three mirrors so that it hits the sample vertically from the top (for a close up view of the wavefront retardation by the cell see Figure 2, which will be discussed in detail further below). Beneath the sample, a microscope objective (MO, 40x, NA 0.75, EC Plan-Neofluar air objective, Carl Zeiss Microscopy GmbH, Göttingen, Deutschland) 


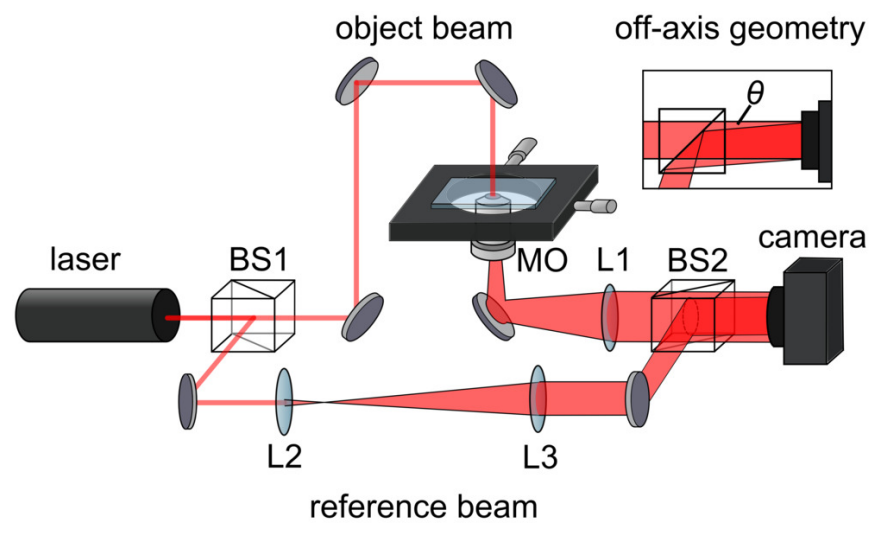

Figure 1: Schematic drawing of the setup of the digital holographic microscope. All parts and their abbreviations are described in the text. The inset shows a top view of the off-axis geometry.

is used to collect the retarded wavefront. Mounting the MO on an axial translation stage (not shown for simplicity) enables focusing. After being focused in the MO the beam is divergent and has a spherical wavefront. This additional spherical phase pattern leads to subsequent difficulties in quantitative analysis. Hence, the divergent beam is collimated by a lens (L1) with a focal length of $200 \mathrm{~mm}$. This leads to the formation of a parallel wavefront and eliminates the spherical phase pattern [14]. To check for proper alignment, a shearing interferometer (Shearing Interferometer, Thorlabs GmbH, Dachau, Germany) can be used for testing the collimation of the beam while aligning the setup. In the reference path a telescope built from two lenses with focal lengths of $100 \mathrm{~mm}$ (L2) and $400 \mathrm{~mm}$ (L3) are used to adapt the beam diameter to the size of the camera chip. Again, the collimation of the reference beam is checked using a shearing interferometer. To be able to adjust the relative intensities of the two beam paths, a filter wheel (continuously variable ND Filter, Thorlabs GmbH, Dachau, Germany) is introduced in the reference path (not shown for clarity). A second beam-splitter cube (BS2) is used to overlap the two beams.Reference and object beam are built to have about the same length between BS1 and BS2. The overlap of the two beams is implemented in a so-called off-axis geometry (see inset in Figure 1). This means that the object and reference beam are not fully aligned on the same optical path behind BS2 but the two beam paths form a small angle $\theta$ (not more than a few degrees), which is important for the analysis. Overlapping the two collimated beams leads to an interference pattern of parallel lines superimposed with an image of the sample from the object path (see Figure $3(\mathrm{~A}))$. The interference pattern is recorded with a CMOS camera placed behind BS2. Since a laser is a coherent light source, it is important to use a camera without a glass cover in front of the chip to avoid additional detrimental interference artifacts. The monochrome CMOS camera used here has 1280x1024 pixels with a pixel size of 5.2 $\mu \mathrm{m}$ (MCE-B013-UW, Mightex Systems, Pleasanton, California, USA). The periodicity of the interference fringes depend on the angle $\theta$ between the overlapping beams. For reasons that will be discussed later in the analysis section the period of the interference pattern has to be small. However, a restriction 
for the period of the interference pattern is given by the pixel size, i.e. the periodicity needs to be well resolved. For the particular setup and camera described here, a period of about 4.4 pixels per line pair which translates to an angle $\theta$ of about $1.6 \mathrm{deg}$ works well. When imaging cells with DHM in this configuration, it is possible to capture more than one cell in one image. However, for the analysis of the cells it is important that they are well separated. For each measurement a background image is recorded additionally by moving the cell slightly out of the field of view with the translation stage. The recorded images are analyzed to obtain a two dimensional phase map of the cell.

\section{Measurement Preparation}

Before starting the measurement, the RI of the medium and the magnification of the microscope have to be determined. A successful measurement of the RI of spherical cells in solution requires a specific sample preparation. The cells need to be attached to a cover slip in order to stay in focus. Additionally, the cells need to be spherical during the measurement so that the height can be determined correctly (see Figure 2). The following protocol serves as a basic guideline for measurement preparations.

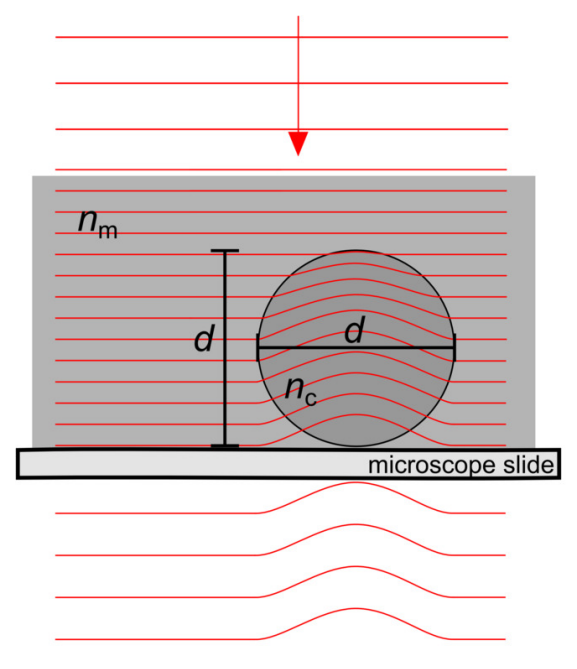

Figure 2: Schematic drawing of a spherical cell on a microscope slide depicting the change in wavefront as it traverses the cell. Here, $d$ is the diameter of the cell, $n_{m}$ is the $\mathrm{RI}$ of the medium and $n_{c}$ the RI of the cell.

\subsection{Materials}

- Slide with micrometer scale (graticule)

- Refractometer (e.g. Abbe-Refractometer 2WAJ, Arcarda GmbH, Reichelsheim, Germany)

- Glass cover slip 
- Poly-L-Lysine (PLL) $0.01 \%$ solution or Poly-D-Lysine (PDL)

- Phosphate-buffered saline (PBS)

- Cells and medium

\subsection{Method}

1. Scale Determination

The magnification of the system can be determined with the help of a graticule. Even though the magnification usually does not change significantly over time, this can be repeated on a regular basis before starting a measurement to consider instabilities due to e.g. temperature drifts.

2. RI of Medium

Before every measurement, the RI of the medium needs to be measured using a refractometer.

3. Cell Preparation

a) Take $2-3 \mathrm{~mL}$ of cells out of the culture dish

b) Centrifuge and resuspend in a medium of choice for a final concentration of approximately $3 \times 10^{5}$ cells $/ \mathrm{mL}$

c) Place $100 \mu \mathrm{L}$ of PLL on the cover slip and let it settle for $10 \mathrm{~min}$

d) Remove PLL and wash twice with PBS

e) Remove PBS from cover slip and add 100-120 $\mu \mathrm{L}$ of cell suspension

f) Wait for $10 \mathrm{~min}$ for the cells to attach to the bottom

g) Carefully remove non-attached cells by pipetting off most of the cell suspension (be careful not to "dry" the cells) and add approximately the same amount of fresh medium

\subsection{Comments}

- Coating the microscope slides with PLL leads to a charge based attachment of cells. For every cell type one has to make sure that the above protocol fulfills the following requirements. The cells have to attach to the microscope slide and must retain their spherical shape without extensively spreading on the microscope slide during the measurement period (about $15 \mathrm{~min}$ ). Spherical shape can be controlled for example by using confocal fluorescence microscopy with a well-calibrated microscope.

- The difference of the RI of the medium to the average RI of the cells should not be less than about 0.001, otherwise the contrast will be too low for a successful analysis. Furthermore, the difference should not be more than about 0.1, otherwise the phase jump at the object-medium interface might not be resolved. These requirements are usually fulfilled for cells in water-based solutions. The upper limit can be reached when measuring artificial objects such as polystyrene beads that, in addition to their high refractive index compared to water, exhibit very sharp boundaries.

- For the measurement of the RI of the medium, an Abbe refractometer with an accuracy of 0.001 is needed. 


\section{Data Analysis}

The goal of the analysis is to determine the RI of an individual cell. In essence, this is done by converting the phase shift information captured in the interference image into a quantitative RI. We implemented the different analysis steps of the algorithm in LabVIEW. A detailed description of the image analysis is presented below (code available upon request).

\subsection{Determination of the Phase Distribution}

The intensity image or hologram (Figure 3(A)) obtained from the DHM is an interference image resulting from the overlap of the reference wavefront $(R)$ and the object wavefront $(O)$. The critical phase information is encoded within the interference pattern. To extract the phase information, we need to consider the intensity $I$ of the overlapping beams:

$$
I=\left|R^{2}\right|+\left|O^{2}\right|+R^{*} O+O^{*} R
$$

where $R^{*}$ and $O^{*}$ are the complex conjugates of the wavefront of the reference beam and the object beam respectively. The first two terms of equation (1) contain information about the amplitude of the individual waves. The last two terms result from the interference of object $O$ and reference $R$ wave. They include the information about phase and amplitude of the object and reference wave. To be able to separate the different terms of the recorded intensity, a Fourier transform is applied. Due to the interference pattern in the off-axis geometry the terms that contain the phase information are shifted away from the origin in Fourier space [15]. In contrast, the first two intensity terms in equation (1) stay at the origin in Fourier space. For a better visualization of the result of the Fourier transform, the absolute value is plotted on a logarithmic scale in Figure 3(B). Here, the first two terms of equation (1) contribute to the peak in the center of Fourier space whereas the last two terms each give rise to one of the side peaks. To improve the image contrast in Fourier space, the central peak is suppressed by subtracting the mean intensity value from each pixel in the raw intensity image prior to the Fourier transform [15]. The phase information of interest is equally encoded in both side peaks and only one of the peaks is required for phase retrieval. For this, a filter is applied in Fourier space that removes all other contributions besides the peak chosen (white circle in Figure 3(B)). We apply a Gaussian filter to reduce ringing artefacts in the reconstructed phase. After filtering, the frequencies are shifted to the center of Fourier space. Subsequently an inverse Fourier transform is applied yielding the complex field $F$ from which the phase can be calculated [15].

$$
\Delta \varphi=\arctan \left(\frac{\Im(F)}{\Re(F)}\right)
$$

The choice of the filter width is important. If the width is too wide, frequencies of the central peak may affect the reconstructed image. If the filter is too small, crucial higher frequencies might be omitted which lowers the resolution of the phase distribution. The positions of the side peaks are dependent on the period of the interference pattern. As discussed earlier, this period is given by the angle $\theta$ of the off-axis geometry. Therefore, the distance from the side peaks to the center of Fourier space can be adjusted by varying the off-axis angle in the setup. Good filtering requires that the side peaks are well separated from the peak at the center of 


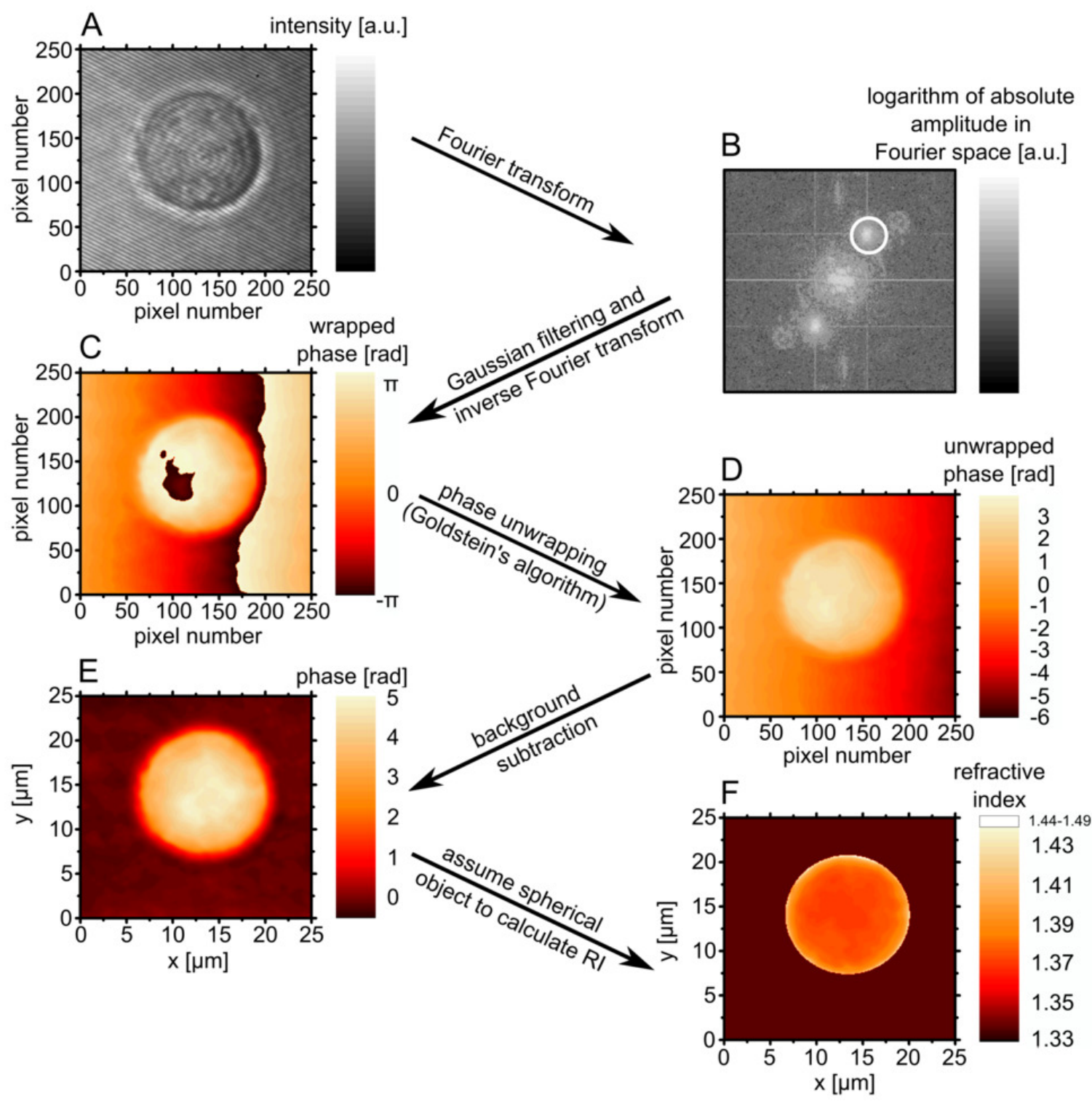

Figure 3: Analysis steps to determine the refractive index (RI) of an HL60 cell from a digital holographic image. (A) Raw intensity image of CMOS camera. (B) Logarithm of absolute value of Fourier transform of the intensity image. The zero frequency component lies in the center of the shown Fourier space image. The white circle indicates the position of the Gaussian filter. (C) Wrapped phase image. (D) Unwrapped phase image. (E) Unwrapped phase with background correction. (F) RI distribution. 
Fourier space. This can be achieved by choosing $\theta$ such that the interference pattern has a small period.

Note that the phase calculated from equation (2) only contains values between $-\pi$ and $\pi$. However, the physical phase shift may contain values outside of this range (modulo $2 \pi$ ). In that case, the computed phase image contains sudden phase jumps of $2 \pi$ (Figure $3(\mathrm{C})$ ) and is termed wrapped phase image. Thus, a crucial part of the analysis is to unwrap the phase. This can be done with the help of Goldstein's algorithm [16]. The underlying principle of the algorithm is to scan for $2 \pi$ phase jumps within the wrapped data and to add an additional phase of $2 \pi$ to the part of the image with the lower phase. A representative image of the unwrapped phase is shown in Figure 3(D).

As discussed earlier, the distance of the side peaks to the center of Fourier space is determined by the angle $\theta$ of the off-axis geometry. If the Gaussian filtered region in Fourier space is not shifted to the exact center before Fourier inversion, the phase image will be superimposed by a linear ramp whose slope is dependent on the angle $\theta$. Since the shift in Fourier space is discrete, the ramp cannot be fully removed, and a correction of the unwrapped image is necessary. There are two ways to accomplish this correction. One possibility is to take a background image as described in the setup section and to subtract the background phase from the measurement phase. Before subtraction, the background image has to be processed exactly the same way as the measurement image. A second possibility is to apply a ramp correction. A linear fit in the $\mathrm{x}$ - and $\mathrm{y}$-direction to regions of the phase image that are known to be background is used to evaluate the slope and the offset of the ramp. This computer generated background image is then subtracted from the unwrapped phase image. The ramp and offset correction can also be applied in series with the background correction in case of marginal drifts between the measurement and background images. Applying both background correction and ramp correction gives the best results. If the setup or the sample do not allow recording an additional background image, a ramp correction alone can be sufficient. After the background correction, one obtains a two dimensional phase distribution of the studied cell, as shown in Figure 3(E).

\subsection{Determination of the refractive index}

The last step is to calculate the average RI from the phase distribution. When light passes through an object with the optical path length $s=h \cdot n$, where $h$ is the thickness and $n$ the RI of the object, it experiences a phase shift $\Delta \varphi$ compared to light that does not pass this object

$$
\Delta \varphi=\frac{2 \pi}{\lambda} \Delta s
$$

where $\Delta s$ is the optical path difference between these two light paths and $\lambda$ is the vacuum wavelength of the light.

In DHM measurements the phase shift due to the cell $\Delta \varphi_{c}(x, y)$ is the quantity of interest. To measure this phase shift, the cell with an axially averaged RI distribution $n_{c}(x, y)$, an effective axial thickness profile $h_{c}(x, y)$ and a diameter $d$ (see Figure 2) is suspended in a medium with a known RI $n_{m}$ and is introduced into the object path of the setup. An additional phase shift is present in the resulting phase image due to an optical path difference $\Delta s_{0}(x, y)$ between object and reference path which results e.g. from the optical components, 
the medium above the cell in the object path or slight differences in the lengths of the reference and object path. The path difference $\Delta s_{0}(x, y)$ is present in every measurement and can be considered as a setup-specific background.

The measured optical path difference between object and reference path includes both the contribution of the cell as well as the background and can be written as:

$$
\frac{\Delta \varphi(x, y) \lambda}{2 \pi}=h_{c}(x, y) n_{c}(x, y)+\left(d-h_{c}(x, y)\right) n_{m}+\Delta s_{0}(x, y)
$$

For reasons of simplicity the dependence of $\Delta \varphi, h_{c}, n_{c}$ and $\Delta s_{0}$ on the coordinated $x$ and $y$ will be omitted in the following. Equation (4) can be rewritten as:

$$
\frac{\Delta \varphi \lambda}{2 \pi}=h_{c} \underbrace{\left(n_{c}-n_{m}\right)}_{\Delta s_{c}}+\underbrace{d n_{m}+\Delta s_{0}}_{\Delta s_{B G}}
$$

Here, the first part on the right side of the equation is the cell-specific optical path difference $\Delta s_{c}$. The second part, termed $\Delta s_{B G}$, is the total background which is subtracted according to the previous section. Inserting $h_{c}=0$ in equation (5) yields the phase of the background image, i.e. with medium but without sample in the light path :

$$
\frac{\Delta \varphi_{B G} \lambda}{2 \pi}=d n_{m}+\Delta s_{0}
$$

Hence, subtracting the phase shift of the background image (equation (6)) from the phase shift measured with the cell in the field of view (equation (5)) yields the cell-specific phase shift $\Delta \varphi_{c}$. Rewriting this difference results in an expression for the axially average refractive index of the cell at each lateral position:

$$
n_{c}=n_{m}+\frac{\Delta \varphi_{c} \lambda}{2 \pi h_{c}}
$$

The only unknown parameter on the right-hand side of equation (7) is the thickness of the cell $h_{c}$. Assuming a spherical cell, the cell's thickness can be calculated from the radius at every point of the cell. The radius is obtained by fitting a circle to the edge of the cell in the phase image. The edges can be determined with an edge detection algorithm, e.g. Canny edge detection (included in LabVIEW). Knowing the thickness of the cell at each position, the integral RI for each pixel within the cell is calculated. The points near the edge are excluded since the thickness at these points approaches zero and the RI becomes ill defined. The RI of the cell is determined as a weighted average, where all individual integral refractive index values within the perimeter of the cell are weighted with $h_{c}$. This emphasizes RI values at positions with a longer optical path length. In order to capture the average RI value of a whole cell population reliably one has to acquire a large enough sample size to obtain a normal distribution of the cells' RI values.

\section{Discussion}

The protocol described so far provides the reader with the basic know-how for building a simple DHM setup that is able to deliver the phase and RI of individual spherical cells. However, there are various possibilities to extend or replace parts of the setup and analysis method which will be discussed in the following sections. 


\subsection{Setup Alterations}

One approach to further enhance digital holographic microscopy is the improvement of the quality of the acquired intensity images and consequently the gathered phase maps. This can be especially of interest if the goal is a detailed analysis of phase or RI changes within a single cell. One possibility in this direction is noise reduction. In the setup described, the laser is used as the light source, which being a source of coherent light, may introduce additional interference patterns due to back reflections at glass interfaces or other obstacles in the beam path. A reduction of this unwanted interference can be achieved using a light source with a shorter coherence length such as a light emitting diode (LED) [17] or by reducing the spatial coherence of a laser with a rotating ground glass in the beam path [18. In comparison to setups that use lasers with a long coherence length, the path length of the reference and the object beam need to be adjusted more precisely for light sources that have shorter coherence lengths. At short coherence length, interference between object and reference beam, which can be used for analysis, requires that the two beam paths have exactly the same length, which therefore requires a more accurate alignment.

There is also the possibility to integrate a system for quantitative phase measurements into commercially available microscopes, rather than building the setup from scratch. The integration of a digital holographic microscope into a commercial microscope is one option [19]. Furthermore, there is the option to buy a digital holographic microscope as a complete system (e.g. Lyncée Tec SA, Lausanne, Switzerland). Alternatively, a camera upgraded with a diffraction grating (SID4-BIO, Phasics S.A., Palaiseau, France) can be used, which uses the principle of quadri-wave lateral shearing interferometry to determine the quantitative phase shift [20]. This technique does not require any changes to the light path of the microscope, and the software acquires the quantitative phase readily; therefore the analysis only requires the determination of the RI from the measured phase.

\subsection{Assumption of Spherical Shape for Refractive Index Determination}

The prerequisite for the analysis method described so far is the assumption that the studied sample must be spherical in shape. In the protocol described here, there is the possibility that, with time, cells spread on the coverslip that they are attached to. Therefore, the validity of the assumption of spherical cells needs to be checked when establishing this technique for a new cell type. Cells often show spherical shapes when they are not attached to any surface but are floating freely in solution. To still be able to control the position of cells in solution for in-focus imaging, an optical trap can be used [21. However, also in this case cells will never resemble a perfect sphere. Furthermore, the recorded phase images contain only the projected phase which limits the analysis in two dimensions. An approach which overcomes these limitations altogether is tomography. Imaging the cell from different angles allows a complete volume reconstruction, such that the thickness can be directly determined from the data. Furthermore, measuring from multiple angles allows computing a full threedimensional RI map of the cell, which permits the unambiguous assignment of the RI of smaller organelles. One possibility of RI tomography involves rotating the sample with a micropipette 22]. Another approach leaves the sample stationary while changing the angle of incidence of the light in the object path by tilting a mirror [23. Sung et al. showed that it is 
possible to acquire three-dimensional phase maps in a microfluidic system [24]. They combine DHM with the synthetic aperture approach to determine three dimensional refractive index maps of single cells.

\subsection{Analysis Alterations}

While we described our analysis method of choice above, there are numerous alternative or enhanced methods. For example it is possible to numerically reconstruct the wave front using a digital reference wave. The digital reference wave resembles the plane wave used during the acquisition of the hologram. In contrast to classical holography, where a plane wave is needed for an optical reconstruction of the sample, in this method a numerical reconstruction is achieved by multiplications of the hologram with a digital reference wave [25]. A further analysis approach allows to numerically refocus the gathered data which is achieved by changing the reconstruction distance [25. This makes focusing possible without mechanically moving the specimen, which is very valuable for imaging dynamic processes. An even further extension is the use of numerical autofocusing of the gathered data. Here, the optimal numerical focus is obtained by maximizing the sharpness of the numerically focused image [26]. The advantage of this method is that only one image needs to be taken and that the autofocusing can be performed computationally in a subsequent step after image acquisition. Yourassowsky and Dubois introduced a microfluidic channel in their setup to image plankton organisms of different sizes and in different focus planes [27. They employed numerical focusing to overcome the problem of different focal planes. Other possibilities of post-processing are methods to improve the image quality. This concept was implemented by Colomb et al. [28]. They were able to overcome aberrations in the imaging system by implementing numerical post-processing. A crucial part of the analysis is the phase unwrapping process. Several alternatives to Goldstein's algorithm exist. One of these possibilities relies on the fact that changing the above mentioned numerical reconstruction distance leads to a spatial shift of the $2 \pi$ phase jumps in the reconstructed field [29]. By computing phase images with multiple reconstruction distances an unwrapped phase image can be obtained.

While the above mentioned alterations correspond to alternatives for the phase extraction, there are also different parameters that can be measured and measurement modalities that can be used in quantitative phase microscopy. For cells that have a nearly uniform RI distribution, e.g. red blood cells, the thickness profile can be determined from the phase [30]. Rappaz et. al compared the volume measurements of red blood cells with DHM to other techniques including volume determination from confocal stacks and measurements performed with an impedance volume analyzer [31. They found that the determined volume of red blood cells with confocal microscopy and DHM gives comparable results. DHM requires the acquisition of only one image to determine the volume of a red blood cell. In comparison, in confocal microscopy a complete z-stack needs to be acquired to fulfill the same task. However, one has to be aware of the fact that the analysis of red blood cells is not representative for the general analysis of cells. The determination of the volume depends on the homogeneity of the refractive index of red bloods cell. This assumption is valid, because red blood cells lack internal structures and are filled with hemoglobin at constant concentration throughout. The determination of the volume using a single DHM image is not possible in the case of large RI variations and an unknown RI distribution. Another application of phase imaging, 
is suitable for cells that are highly dynamic, e.g. contracting cardiomyocytes, here temporal measurements can be used to characterize their cell dynamics [32. A further example involves the determination of the dry mass of cells [33]. All these numerous alternatives and add-ons show the diverse ways in which data acquisition and analysis can be adjusted to meet specific measurement goals.

\section{Summary}

This chapter gives an overview of all the steps needed for the implementation of digital holographic microscopy. The setup, sample preparation and analysis routines are described in detail and allow for an easy but robust determination of the phase and RI map of spherical cells. We also discuss further extensions to the proposed setup and present a brief overview of the diverse biological applications. The methods described in this chapter form the basis for versatile applications in biological studies.

\section{References}

[1] N. Pavillon, J. Kühn, C. Moratal, P. Jourdain, C. Depeursinge, P. J. Magistretti, and P. Marquet, "Early cell death detection with digital holographic microscopy," PLoS ONE, vol. 7, p. e30912, jan 2012.

[2] K. J. Chalut, A. E. Ekpenyong, W. L. Clegg, I. C. Melhuish, and J. Guck, "Quantifying cellular differentiation by physical phenotype using digital holographic microscopy," Integrative biology quantitative biosciences from nano to macro, vol. 4, pp. 280-4, mar 2012 .

[3] A. E. Ekpenyong, S. M. Man, S. Achouri, C. E. Bryant, J. Guck, and K. J. Chalut, "Bacterial infection of macrophages induces decrease in refractive index," Journal of Biophotonics, vol. 6, pp. 393-397, aug 2012.

[4] J. Guck, S. Schinkinger, B. Lincoln, F. Wottawah, S. Ebert, M. Romeyke, D. Lenz, H. M. Erickson, R. Ananthakrishnan, D. Mitchell, J. Käs, S. Ulvick, and C. Bilby, "Optical deformability as an inherent cell marker for testing malignant transformation and metastatic competence," Biophysical journal, vol. 88, pp. 3689-98, may 2005.

[5] R. Drezek, M. Guillaud, T. Collier, I. Boiko, A. Malpica, C. Macaulay, M. Follen, and R. R. Richards-Kortum, "Light scattering from cervical cells throughout neoplastic progression: influence of nuclear morphology, DNA content, and chromatin texture," Journal of Biomedical Optics, vol. 8, no. 1, p. 7, 2003.

[6] L. T. Perelman, V. Backman, M. Wallace, G. Zonios, R. Manoharan, A. Nusrat, S. Shields, M. Seiler, C. Lima, T. Hamano, I. Itzkan, J. V. Dam, J. M. Crawford, and M. S. Feld, "Observation of periodic fine structure in reflectance from biological tissue: A new technique for measuring nuclear size distribution," Physical Review Letters, vol. 80, pp. 627-630, jan 1998.

[7] I. Solovei, M. Kreysing, C. Lanctôt, S. Kösem, L. Peichl, T. Cremer, J. Guck, and B. Joffe, "Nuclear architecture of rod photoreceptor cells adapts to vision in mammalian evolution," Cell, vol. 137, pp. 356-68, apr 2009. 
[8] Z. Blaszczak, M. Kreysing, and J. Guck, "Direct observation of light focusing by single photoreceptor cell nuclei," Optics Express, vol. 22, no. 9, pp. 11043-11060, 2014.

[9] R. Barer and S. Joseph, "Refractometry of living cells part I. basic principles," Quarterly Journal of Microscopical Science, vol. 171, no. 2, pp. 38P-39P, 1954.

[10] B. Rappaz, P. Marquet, E. Cuche, Y. Emery, C. Depeursinge, and P. Magistretti, "Measurement of the integral refractive index and dynamic cell morphometry of living cells with digital holographic microscopy," Optics Express, vol. 13, no. 23, pp. 9361-9373, 2005 .

[11] B. Rappaz, F. Charrière, C. Depeursinge, P. J. Magistretti, and P. Marquet, "Simultaneous cell morphometry and refractive index measurement with dual-wavelength digital holographic microscopy and dye-enhanced dispersion of perfusion medium," Optics Letters, vol. 33, p. 744, mar 2008.

[12] C. L. Curl, C. J. Bellair, T. Harris, B. E. Allman, P. J. Harris, A. G. Stewart, A. Roberts, K. A. Nugent, and L. M. D. Delbridge, "Refractive index measurement in viable cells using quantitative phase-amplitude microscopy and confocal microscopy," Cytometry Part $A$, vol. 65A, no. 1, pp. 88-92, 2005.

[13] B. Kemper, S. Kosmeier, P. Langehanenberg, G. von Bally, I. Bredebusch, W. Domschke, and J. Schnekenburger, "Integral refractive index determination of living suspension cells by multifocus digital holographic phase contrast microscopy.," Journal of Biomedical Optics, vol. 12, no. 5, p. 054009, 2007.

[14] E. Sánchez-Ortiga, P. Ferraro, M. Martínez-Corral, G. Saavedra, and A. Doblas, "Digital holographic microscopy with pure-optical spherical phase compensation," Journal of the Optical Society of America A, vol. 28, no. 7, pp. 1410-1417, 2011.

[15] E. Cuche, P. Marquet, and C. Depeursinge, "Spatial filtering for zero-order and twinimage elimination in digital off-axis holography," Applied Optics, vol. 39, no. 23, pp. 40704075, 2000.

[16] D. C. Ghiglia and M. D. Pritt, Two-dimensional phase unwrapping: theory, algorithms, and software. Wiley-Interscience, 1998.

[17] B. Kemper, S. Stürwald, C. Remmersmann, P. Langehanenberg, and G. von Bally, "Characterisation of light emitting diodes (LEDs) for application in digital holographic microscopy for inspection of micro and nanostructured surfaces," Optics and Lasers in Engineering, vol. 46, pp. 499-507, jul 2008.

[18] F. Dubois, M.-L. N. Requena, C. Minetti, O. Monnom, and E. Istasse, "Partial spatial coherence effects in digital holographic microscopy with a laser source," Applied Optics, vol. 43, p. 1131, feb 2004.

[19] B. Kemper, D. Carl, A. Höink, G. von Bally, I. Bredebusch, and J. Schnekenburger, "Modular digital holographic microscopy system for marker free quantitative phase contrast imaging of living cells," in Biophotonics and New Therapy Frontiers (R. Grzymala and O. Haeberle, eds.), SPIE, apr 2006.

[20] P. Bon, G. Maucort, B. Wattellier, and S. Monneret, "Quadriwave lateral shearing interferometry for quantitative phase microscopy of living cells," Optics Express, vol. 17, no. 15, pp. 13080-13094, 2009. 
[21] B. Kemper, P. Langehanenberg, A. Höink, G. von Bally, F. Wottowah, S. Schinkinger, J. Guck, J. Käs, I. Bredebusch, J. Schnekenburger, and K. Schütze, "Monitoring of laser micromanipulated optically trapped cells by digital holographic microscopy," Journal of Biophotonics, vol. 3, pp. 425-431, jun 2010.

[22] I. Bergoënd, C. Arfire, N. Pavillon, and C. Depeursinge, "Diffraction tomography for biological cells imaging using digital holographic microscopy," in Laser Applications in Life Sciences (M. Kinnunen and R. Myllylä, eds.), SPIE, jun 2010.

[23] W. Choi, C. Fang-Yen, K. Badizadegan, S. Oh, N. Lue, R. R. Dasari, and M. S. Feld, "Tomographic phase microscopy," Nature Methods, vol. 4, pp. 717-719, sep 2007.

[24] Y. Sung, N. Lue, B. Hamza, J. Martel, D. Irimia, R. R. Dasari, W. Choi, Z. Yaqoob, and P. So, "Three-dimensional holographic refractive-index measurement of continuously flowing cells in a microfluidic channel," Physical Review Applied, vol. 1, p. 14002, feb 2014.

[25] E. Cuche, P. Marquet, and C. Depeursinge, "Simultaneous amplitude-contrast and quantitative phase-contrast microscopy by numerical reconstruction of fresnel off-axis holograms," Applied Optics, vol. 38, no. 34, pp. 6994-7001, 1999.

[26] P. Langehanenberg, B. Kemper, D. Dirksen, and G. von Bally, "Autofocusing in digital holographic phase contrast microscopy on pure phase objects for live cell imaging," Applied Optics, vol. 47, pp. D176-D182, jul 2008.

[27] C. Yourassowsky and F. Dubois, "High throughput holographic imaging-in-flow for the analysis of a wide plankton size range," Optics Express, vol. 22, p. 6661, mar 2014.

[28] T. Colomb, E. Cuche, F. Charrière, J. Kühn, N. Aspert, F. Montfort, P. Marquet, and C. Depeursinge, "Automatic procedure for aberration compensation in digital holographic microscopy and applications to specimen shape compensation," Applied Optics, vol. 45, no. 5, pp. 851-863, 2006.

[29] A. Khmaladze, T. Epstein, and Z. Chen, "Phase unwrapping by varying the reconstruction distance in digital holographic microscopy," Optics Letters, vol. 35, no. 7, pp. 10401042, 2010.

[30] G. Popescu, T. Ikeda, C. a. Best, K. Badizadegan, R. R. Dasari, and M. S. Feld, "Erythrocyte structure and dynamics quantified by hilbert phase microscopy," Journal of Biomedical Optics, vol. 10, no. 6, p. 060503, 2013.

[31] B. Rappaz, A. Barbul, Y. Emery, R. Korenstein, C. Depeursinge, P. J. Magistretti, and P. Marquet, "Comparative study of human erythrocytes by digital holographic microscopy, confocal microscopy, and impedance volume analyzer," Cytometry Part A, vol. $73 \mathrm{~A}$, pp. 895-903, oct 2008.

[32] N. T. Shaked, L. L. Satterwhite, N. Bursac, and A. Wax, "Whole-cell-analysis of live cardiomyocytes using wide-field interferometric phase microscopy," Biomedical Optics Express, vol. 1, p. 706, aug 2010.

[33] T. A. Zangle, D. Burnes, C. Mathis, O. N. Witte, and M. A. Teitell, "Quantifying biomass changes of single CD8+ T cells during antigen specific cytotoxicity," PLoS ONE, vol. 8, p. e68916, jul 2013. 